\title{
A case of follicular carcinoma of thyroid with renal metastasis
}

\author{
SK Kodisinghe ${ }^{1}$, SCR Perera ${ }^{2}$ \\ ${ }^{1}$ Senior House Officer, ${ }^{2}$ Consultant Oncological Surgeon, Oncosurgical Unit, Teaching Hospital, Karapitiya, \\ Galle.
}

e-mail address of the corresponding author, Dr. S Kuleesha Kodisinghe: skkodisinghe@yahoo.com

\section{Introduction}

Thyroid carcinoma comprises $1 \%$ of all malignancies. It is the commonest malignant endocrine tumour'. Most thyroid carcinomas arise from the follicular epithelium and are differentiated carcinomas.

The two main groups are papillary $(80 \%)$ and follicular $(10-15 \%)^{2}$. Follicular carcinoma has a predilection for spreading via the bloodstream early, and metastasis is most commonly to the lung and bone ${ }^{1}$. So far only about 20 cases of differentiated thyroid carcinoma metastasising to the kidney have been reported. We report a rare case of follicular thyroid carcinoma metastasising to the kidney.

\section{Case report}

A 61 year-old woman had a gradually enlarging goitre and a large scalp lump in the occipital region for 4 years. There was no hoarseness or dysphagia and she was euthyroid. On examination, there was a large goitre displacing the trachea to the right and a soft boggy mass over the occipital region of the scalp. MRI brain showed a soft tissue lesion eroding the occipito-parietal bone and the bony defect was evident on the skull x-ray (Figure 1). FNAC of the thyroid showed follicular neoplasm. The chest $\mathrm{x}$-ray showed multiple lung metastases. CT scan of the chest and abdomen confirmed the lung metastases, and also incidentally detected a tumour in the upper pole of the left kidney (Figure 2). Serum thyroglobulin level was markedly elevated $(>100,000 \mathrm{ng} / \mathrm{mL})$.

Total thyroidectomy and left radical nephrectomy was performed, during which a large tumour arising from the left lobe of the thyroid, a tumour arising from the upper pole of the left kidney and suspicious peritoneal deposits on appendices epiploicae were found. Histopathology showed an invasive follicular carcinoma of thyroid with metastatic deposits in the left kidney and appendices epiploicae. After a successful post-operative recovery, the patient is to be followed up by the oncologist.

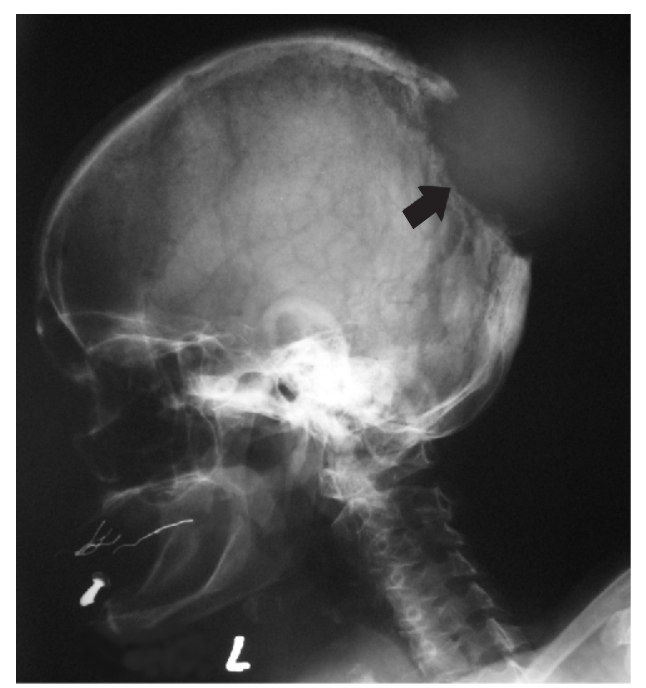

Figure 1 - Skull $x$-ray showing the bony defect (arrow).

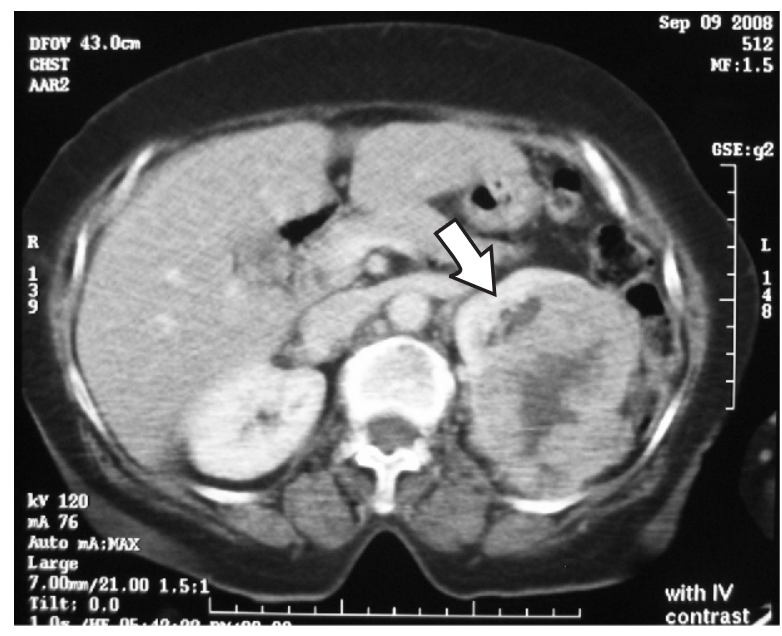

Figure 2 - Contrast enhanced CT scan of the abdomen showing the tumour in the upper pole of left kidney (arrow). 


\section{Discussion}

Both follicular adenoma and carcinoma show similar histological features and demonstration of capsular or vascular invasion is essential for the diagnosis of carcinoma. This is not possible by FNAC, and histological examination of the resected specimen is needed ${ }^{1}$.

Treatment of the primary tumour is either total thyroidectomy or lobectomy followed later by completion thyroidectomy depending on the definitive histology $y^{1,3}$.

Radioiodine therapy is used to treat metastatic disease in patients with differentiated thyroid cancer ${ }^{4}$. Surgical removal of resectable metastases can be a valuable complement to this therapy, and the complicated surgical treatment of metastases is justified by the favorable effect it has on prognosis and quality of life 5 . Bone metastases are generally not curable by radioiodine therapy alone and external beam radiotherapy may have to be considered ${ }^{3}$.

This patient has undergone maximal possible debulking of malignant tissue by total thyroidectomy and left nephrectomy. She will need radioiodine therapy for ablation of residual neoplastic tissue in the thyroid bed and left renal bed and metastatic deposits in lungs and bone. She will also require external beam radiotherapy for the bony metastasis of the skull.

\section{References}

1. Balasubramanian SP, Thomas WEG. Thyroid neoplasms (including the solitary nodule). Surgery 2007; 25: 482-6.

2. Anne Marie McNicol. Pathology of thyroid tumours. Surgery 2007; 25: 458-62.

3. Guidelines for the management of thyroid cancer, $2^{\text {nd }}$ edition. British Thyroid Association, Royal College of Physicians. 2007.

4. Maxon HR. Quantitative radioiodine therapy in the treatment of differentiated thyroid cancer. Quarterly Journal of Nuclear Medicine 1999; 43: 313-23.

5. Niederle B, Roka R, Schemper M, Fritsch A, Weissel M, Ramach W. Surgical treatment of distant metastases in differentiated thyroid cancer: Indication and results. Surgery 1986; 100: 1088-97. 